\title{
The HvDOF19 transcription factor mediates the abscisic acid-dependent repression of hydrolase genes in germinating barley aleurone
}

\author{
Miguel Ángel Moreno-Risueño, Isabel Díaz, Laura Carrillo, Rebeca Fuentes \\ and Pilar Carbonero
}

\begin{abstract}
Summary
Upon germination of seed cereals, mobilization of the reserves stored in the endosperm is regulated by the phytohormones gibberellins (GA) and abscisic acid (ABA). In barley, the cis regulatory elements and the transacting factors mediating the ABA response of hydrolase genes remain elusive. Two new barley genes, HvDof17 and HvDof 19, encoding transcription factors (TFs) of the DNA binding with one finger (DOF) class have been characterized and their role upon germination investigated. HvDOF19 binds in a specific manner to the pyrimidine box within the GARC of a thiol-protease gene (A/21), and mediates the ABA repression of this gene in the barley aleurone. Silencing of HvDof19 in transient expression assays diminishes the inhibitory effect of ABA upon expression of the Al21 gene promoter. Transcripts from HvDof17 and HvDof19 accumulate early in germinating aleurones with a peak at $16 \mathrm{~h}$ after seed imbibition (hai), whereas the mRNAs of the GA-induced activator GAMYB remain little expressed. At 48 hai, mRNA content of both genes is comparatively insignificant compared with that of GAMYB, which reaches a maximum. Both TFs repress, in transient expression assays, the GA- and the GAMYB-mediated activation of this thiol-protease gene (Al21). In addition, HvDOF17 and HvDOF19 interact with GAMYB in plant cell nuclei, and HvDOF17, but not HvDOF19, interferes with the DNA binding of GAMYB to its target site in the promoter of the Al21 gene. A regulatory model of hydrolase gene expression upon germination is proposed.
\end{abstract}

Keywords: abscisic acid-induced DOF, barley seeds, gibberellin-repressed DOFs, germinating aleurone, HvDOF19, HvDOF17.

Introduction

The hormonal balance between gibberellins (GA) and abscisic acid (ABA) is a crucial factor in seed germination, although other phytohormones such as brassinosteroids and ethylene, as well as light and sugars, also influence this physiological process (Aoki et al., 2006; Brocard-Gifford et al., 2003; Lovegrove and Hooley, 2000; Steber and McCourt, 2001). ABA is involved in the maintenance of dormancy, inhibits germination and is required in the glucose-mediated developmental arrest during post-germination (Arenas-Huertero et al., 2000; Gubler et al., 2005; Lopez-Molina et al., 2001, 2002). ABA perception seems to occur both at the plasma membrane and in the cytoplasm, and it has been suggested that different receptors might perceive this hormone in different response pathways (Allan et al., 1994; Finkelstein et al., 2002). In Arabidopsis, an ABA receptor (FCA) involved in RNA metabolism and in the control of flowering time has been identified (Razem et al., 2006). In barley, the protein ABAP1 could be an ABA receptor located in the plasma membrane of the aleurone cells (Razem et al., 2004). Genetic approaches have led to the characterization of several intermediates of the ABA signal transduction pathway. However, a complete identification of the network components is still lacking (Finkelstein et al., 2002).

In cereals, imbibition triggers a decrease in ABA seed content and promotes the synthesis of GA by the embryo that diffuses to the aleurone (Jacobsen et al., 2002; Kaneko et al., 2002). In this tissue, GA is perceived by a soluble 
receptor promoting the degradation of the DELLA proteins that repress the GA response (Ueguchi-Tanaka et al., 2005). In addition, this hormone could be perceived by an unknown membrane-localized GA receptor. This would trigger a signal transduction pathway involving increases in cytoplasmic calcium and in $\mathrm{pH}$ values, as well as increases in heterotrimeric $\mathrm{G}$ proteins, protein kinases and Ser/Thr O-linked $\mathrm{N}$-actylglucosamine transferases (Gilroy and Jones, 1994; Hartweck and Olszewski, 2006; Jacobsen et al., 1995; McCubbin et al., 2004; Robertson et al., 1998; Sun and Gubler, 2004). Eventually, transcription of genes encoding hydrolases is activated. These enzymes, mainly $\alpha$-amylases and proteases, are secreted into the endosperm where they mobilize stored reserves, releasing the nutrients that will support embryo growth until photosynthesis is fully established (Eastmond and Russell, 2005). Most of GA effects can be antagonized by ABA. In dormant seeds and under unfavourable germinating conditions, ABA suppresses GA-induced expression of hydrolase genes (Gomez-Cadenas et al., 2001b; Lovegrove and Hooley, 2000).

Functional analyses of the promoters of GA-responsive hydrolase genes in the barley aleurone (Jacobsen et al., 1995) have identified a conserved cis-acting response complex, the GARC. This complex comprises three motifs necessary for a full GA response: (1) the $5^{\prime}$-TAACAA/GA-3 box or GA-responsive element (GARE); (2) the pyrimidine box, 5'-C/TCTTTTC/T-3'; and (3) the 5'-TATCCAC-3' element. In addition, in low-pl $\alpha$-amylase genes, a putative Opaque-2 binding sequence (O2S: $5^{\prime}$-CTTGACCATCATC- $3^{\prime}$ ) is also involved in the GA response (Gomez-Cadenas et al., 2001a; Lanahan et al., 1992; Rogers et al., 1994). However, the cis-motifs responsible for the ABA repression of these hydrolase genes have not yet been identified.

Over the past few years, several transcription factors (TFs) have been demonstrated to interact with the three motifs of the GARC. In barley, these TFs belong to the R2R3MYB, R1MYB and DOF classes (Gubler et al., 1995; IsabelLaMoneda et al., 2003; Mena et al., 2002; Rubio-Somoza et al., 2006a,b). In rice, TFs of the WRKY class have been also described as regulators of hydrolase gene expression through binding to the O2S motif (Xie et al., 2006; Zhang et al., 2004).

The GAMYB protein of the R2R3MYB class, which is GA induced, interacts with the GARE and trans-activates a number of hydrolase gene promoters downstream of the barley DELLA protein SLN1 (Gubler et al., 1995, 1999, 2002; Sun and Gubler, 2004). SLN1 is an indispensable intermediate of GA signaling, and its silencing leads to derepression of $\alpha$-amylase gene expression in aleurone cells (Gubler et al., 2002; Zentella et al., 2002). This derepression is suppressed by exogenous ABA, so it has been suggested that molecular interaction between the signaling cascades mediated by GA and ABA occurs midway in the pathway that leads to the synthesis of hydrolytic enzymes (Zentella et al., 2002). The barley PKABA1 protein kinase, which is $A B A$ induced, participates in the $A B A$ signaling cascade in aleurone cells. Its transient overexpression represses the GA induction of GAMYb (Gomez-Cadenas et al., 2001b), and consequently represses hydrolase gene expression (GomezCadenas et al., 1999; Shen et al., 2001). However, silencing of PKABA1 seems not to affect the ABA repression of $\alpha$-amylase genes (Zentella et al., 2002).

TFs of the DOF class, BPBF and SAD, regulate hydrolase gene expression in germinating barley aleurones through binding to the pyrimidine box of the GARC. They have antagonistic functions. BPBF is a transcriptional repressor whereas SAD is a transcriptional activator, and both interact with GAMYB in cell nuclei (Diaz et al., 2005; IsabelLaMoneda et al., 2003; Mena et al., 2002).

This work deals with the molecular characterization of two novel genes encoding DOF proteins from barley, HvDof19 and HvDof17. These genes are highly expressed in aleurone cells early after seed imbibition (up to $16 \mathrm{~h}$ after seed imbibition; 16 hai), and their mRNAs decrease thereafter. Both TFs are repressors of the GA-induced cathepsin B-like thiol protease gene $A / 21$, and they interact with GAMYB in plant cell nuclei. Whereas HvDof19 is induced by ABA, HvDof17 is not, although both genes are GA repressed. In addition, the HvDof19 RNAi construct diminishes the inhibitory effect of $A B A$ upon the expression of the Al21 gene promoter in transient expression assays, indicating that this factor is necessary for the ABA repression of the GA signaling in aleurone cells. Possible physiological implications are discussed.

\section{Results}

\section{Molecular characterization of new DOF genes from barley}

We had previously reported the role of BPBF and SAD (HvDof24-Pbf and HvDof23-Sad genes, respectively) as regulators of hydrolase gene expression in aleurone cells upon barley seed germination (Isabel-LaMoneda et al., 2003; Mena et al., 2002). Therefore, we decided to explore whether other DOF proteins could also participate in this transcriptional network. As five DOF proteins from rice had been identified as regulators of the expression of a peptidase gene following germination (Washio, 2001), and in our annotation of 26 different DOF genes from barley we had inferred their phylogenetic relationships with genes from rice (MorenoRisueno et al., 2007), we focused on the barley putative orthologs of these five DOF genes from rice. Four clear couples of barley-rice orthologs were: HvDof8-Os02g49440, HvDof2-Os02g45200, HvDof23-Sad-Os04g47990 and HvDof24-

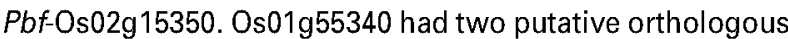
genes in barley, the HvDof19 and HvDof17 genes. 
The expression of these six DOF genes in barley aleurones during an early phase of seed germination and post-germination was explored by real time quantitative PCR (RT-qPCR). In our growing conditions, radicle emerges at 18 hai $(n=200$, $84.2 \pm 5.1 \%$ ) indicating the beginning of the post-germination stage. At different times after water imbibition $(8,12,18$, 24,36 and 48 hai), aleurones were separated from the rest of the seed and collected. Samples at 8,12 and 18 hai (germination) and at 24, 36 and 48 hai (post-germination) were mixed in a 1:1:1 ratio. The results, expressed as relative content of the HvActin2 mRNA levels, indicated that the six barley DOF genes were expressed during the germination stage (Figure 1a). During post-germination, the most abundant transcripts were those of HvDof 19, followed by HvDof24Pbf and HvDof17, and the lowest levels corresponded to HvDof2, followed by HvDof23-Sad and HvDof8 (Figure 1a). When the transcript content of these genes was analogously analyzed by RT-qPCR in germinating embryos collected at the same times as the aleurones, we observed that they were comparatively less expressed (data not shown). Based on this expression pattern and considering that we had already studied the HvDof24-Pbf gene, the HvDof19 and HvDof17 genes were selected for further characterization.

The nucleotide and deduced amino acid sequences of the cDNAs of HvDof19 and HvDof17 showed that both genes encoded for putative proteins of 201 and 215 residues, with a predicted molecular mass of 21.9 and $23.0 \mathrm{kDa}$, respectively. Their DOF domains shared $89 / 98 \%$ of identical/similar residues, whereas along the protein, the identity/similarity percentage dropped to $41 / 49 \%$. Neither of the two genes had introns within their open reading frames (ORFs) (MorenoRisueno et al., 2007).

The expression of the HvDof19 and HvDof17 genes in barley aleurone layers is hormonally regulated

A more detailed expression pattern of the HvDof19 and HvDof17 genes in germinating barley aleurones at $8,16,24$, 36,48 and 72 hai was investigated by RT-qPCR. As shown in Figure $1(\mathrm{~b})$, a rapid increase in the mRNA accumulation of the HvDof17 and HvDof19 genes that peaked at 16 hai was detected. However, at 24 hai the HvDof 19 transcripts had fallen to the same levels detected at 8 hai, whereas the HvDof17 mRNAs decreased only by $25 \%$. In contrast, the mRNAs levels of the gene encoding a GA-responsive cathepsin B-like protease (Martinez et al., 2003) exponentially increased between 16 and 24 hai. They continued to rise until 48 hai and declined thereafter. The expression pattern of the HvDof24-Pbf gene, previously described as a repressor of hydrolase genes in aleurone (Mena et al., 2002), resembled that obtained for HvDof17 and HvDof19, whereas the mRNAs of the GA-induced GAMyb gene encoding a potent activator of the same hydrolase genes (Gubler et al., 1999) increased to a maximum at 48 hai.

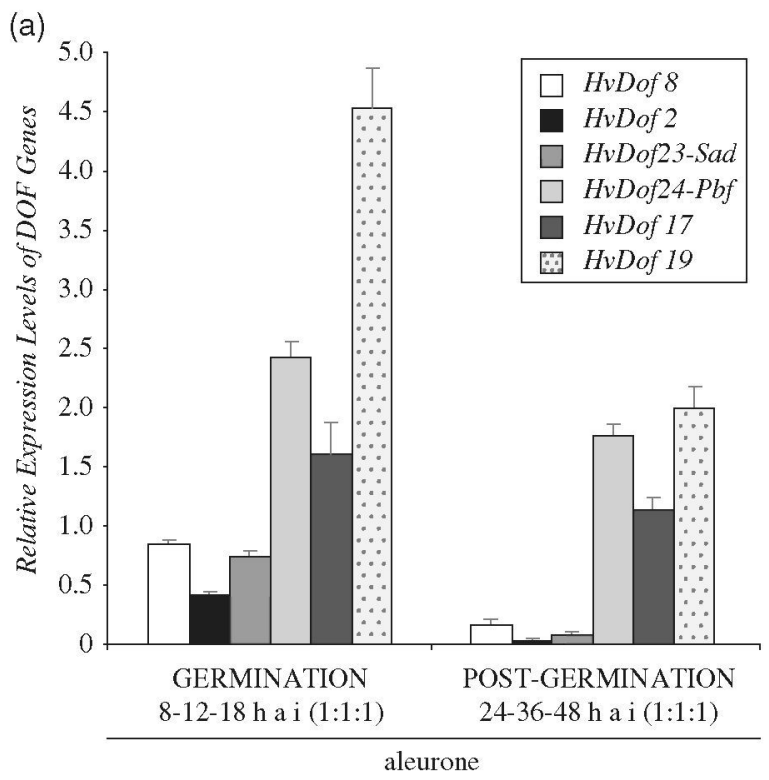

(b)

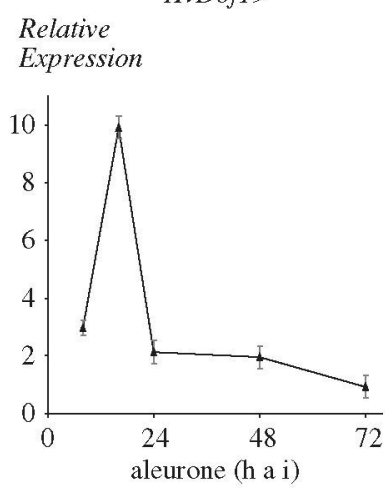

Relative

Expression

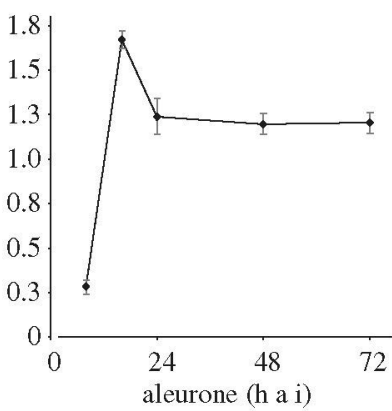

HvDof 24-Pbf

Relative

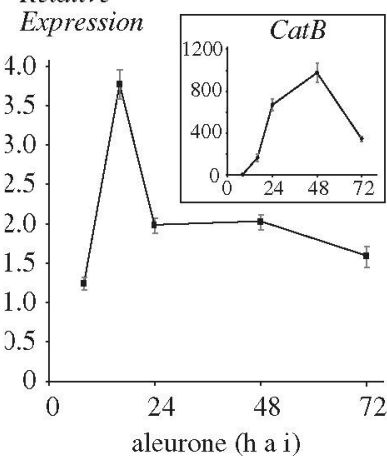

$G A M y b$

\section{Relative}

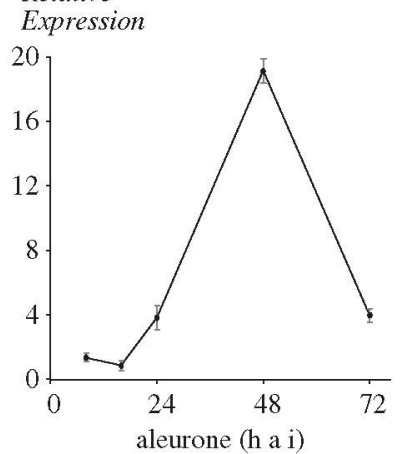

Figure 1. Real time quantitative PCR analysis (RT-qPCR) of several DOF, $G A M y b$, and cathepsin B-like encoding CatB genes.

(a) Relative mRNA content of HvDof2, HvDof8, HvDof77, HvDof79, HvDof23 $\mathrm{Sad}$ and HvDof24-Pbf genes in aleurones of imbibed barley seeds (samples collected at 8,12 and $18 \mathrm{~h}$ after imbibition, hai, were mixed in a ratio $1: 1: 1)$ and in aleurones of post-germinating seeds $(24,36$ and 48 hai; mixture $1: 1: 1)$.

(b) Variation of transcript accumulation of HvDof17, HvDof19, Pbf, GAMyb and $C a t B$ genes in barley aleurones at $8,16,24,48$ and 72 hai. Data were standardized to the barley Actin2 mRNA levels. 
To determine the spatial expression of HvDof17 and HvDof19 within germinating seeds, mRNA in situ hybridization assays were carried out. Samples at 16 hai were hybridized to specific antisense probes for HvDof17 and HvDof19. We detected a strong signal in aleurone cells and in the embryo (Figure 2a). The mRNA label for HvDof17 was mainly observed in the foliar primordia and in the scutellum, whereas for HvDof19 it was prominent in the radicle. As expected, when the samples were hybridized with the sense probes, used as negative controls, no signal above background was detected (Figure 2a).

The accumulation pattern and spatial expression of the HvDof19 and HvDof17 transcripts upon barley seed imbibition suggested that it might be hormonally regulated. To test this possibility, isolated aleurone layers from imbibed de-embryonated seeds were incubated for $20 \mathrm{~h}$ with $1 \mu \mathrm{m}$ $\mathrm{GA}_{3}, 10 \mu \mathrm{M} A B A$ or a mixture of both hormones. Their transcripts were quantified by RT-qPCR. As shown in Figure 2(b), a decrease of 50\% in HvDof 19 and HvDof 17 expression levels was detected with the GA treatment, whereas the incubation with $A B A$ produced an increase of approximately sixfold in HvDof19 mRNAs and it had no effect on the HvDof17 transcript accumulation. Moreover, the enhancer effect of $A B A$ in HvDof19 expression was only slightly decreased by the incubation with the mixture of $G A+A B A$.
As expected from previous data, the cathepsin B-like encoding gene in aleurone layers was induced by GA and repressed by ABA (Martinez et al., 2003). However, HvDof24-Pbf, although induced by GA, was not affected by ABA (Mena et al., 2002).

HvDOF19 and HvDOF17 proteins are localized to the aleurone cell nuclei

To investigate the subcellular localization of HvDOF19 and HvDOF17 proteins, translational fusions of their corresponding ORFs to the GFP were made. These constructs were under the control of the $35 \mathrm{~S}$ promoter followed by the first intron of the maize alcohol dehydrogenase I (Adh) gene.

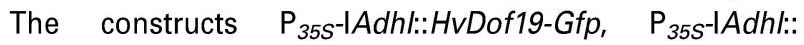
HvDof17-Gfp and the control construct $\mathrm{P}_{35 s^{-}}$Adhl::Gfp were used to transiently transform barley aleurone layers by particle bombardment. After $24 \mathrm{~h}$ of incubation, samples were observed under a confocal microscope. As shown in Figure 3, when the aleurone layers were transformed with the constructs carrying either HvDOF19 or HvDOF17 proteins fused to the GFP, fluorescence corresponding to the emission spectrum of this protein was detected within the cell nucleus (Figure 3a-f). When the aleurones were transiently transformed with $\mathrm{P}_{35 S^{-}} \mid A d h l:: G f p$, the GFP fluorescence
Figure 2. Expression of HvDof19 and HvDof17 in germinating barley seeds and their response to hormonal treatments in aleurone cells.

(a) Spatial expression of HvDof19 and HvDof17 genes in barley seeds (16 hai) determined by mRNA in situ hybridization assays. Upper panels correspond to transversal sections of germinating aleurones and lower panels correspond to longitudinal sections of germinating embryos. Hybridization was performed on the sense and antisense probes as indicated. a, aleurone; en, endosperm; $f p$, foliar primordia; pe, pericarp; $r$, radicle; sc, scutellum; t, testa. Scale bars: $100 \mu \mathrm{m}$. (b) Analyses of the mRNA variation (RT-qPCR) of the HvDof19, HvDof17, HvDof24-Pbf and CatB genes in aleurone layers after $20 \mathrm{~h}$ of incubation in the presence of $1 \mu \mathrm{M} \mathrm{GA}_{3}, 10 \mu \mathrm{m} \mathrm{ABA}$ or a mixture of both hormones. Results were referred to the control value (without hormones) in each case, which was arbitrarily set to 1 . (a)

$H v D o f 19$
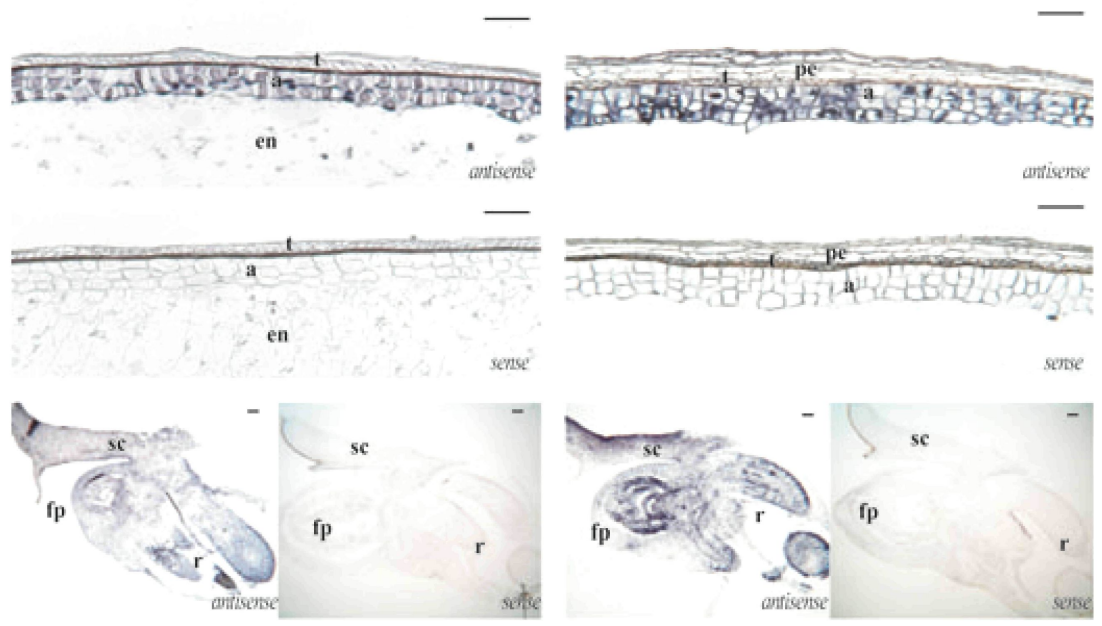

(b)
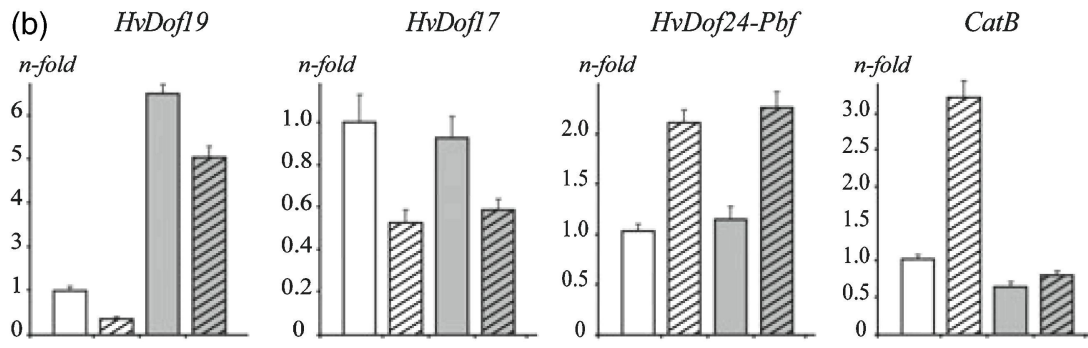

$+\mathrm{ABA} 10 \mu \mathrm{m}$
HvDof17
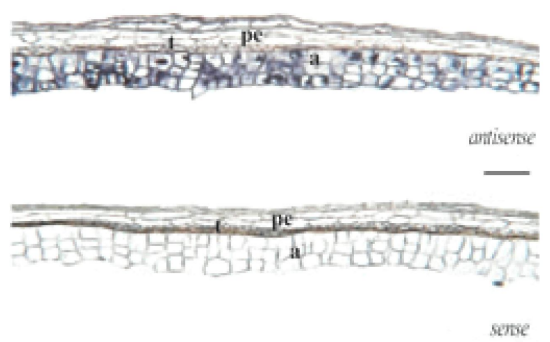

$\square$ Without treatment

$\square+\mathrm{GA} 1 \mu \mathrm{m}$

$\square+\mathrm{GA} 1 \mu \mathrm{m}+\mathrm{ABA} 10 \mu \mathrm{m}$ 

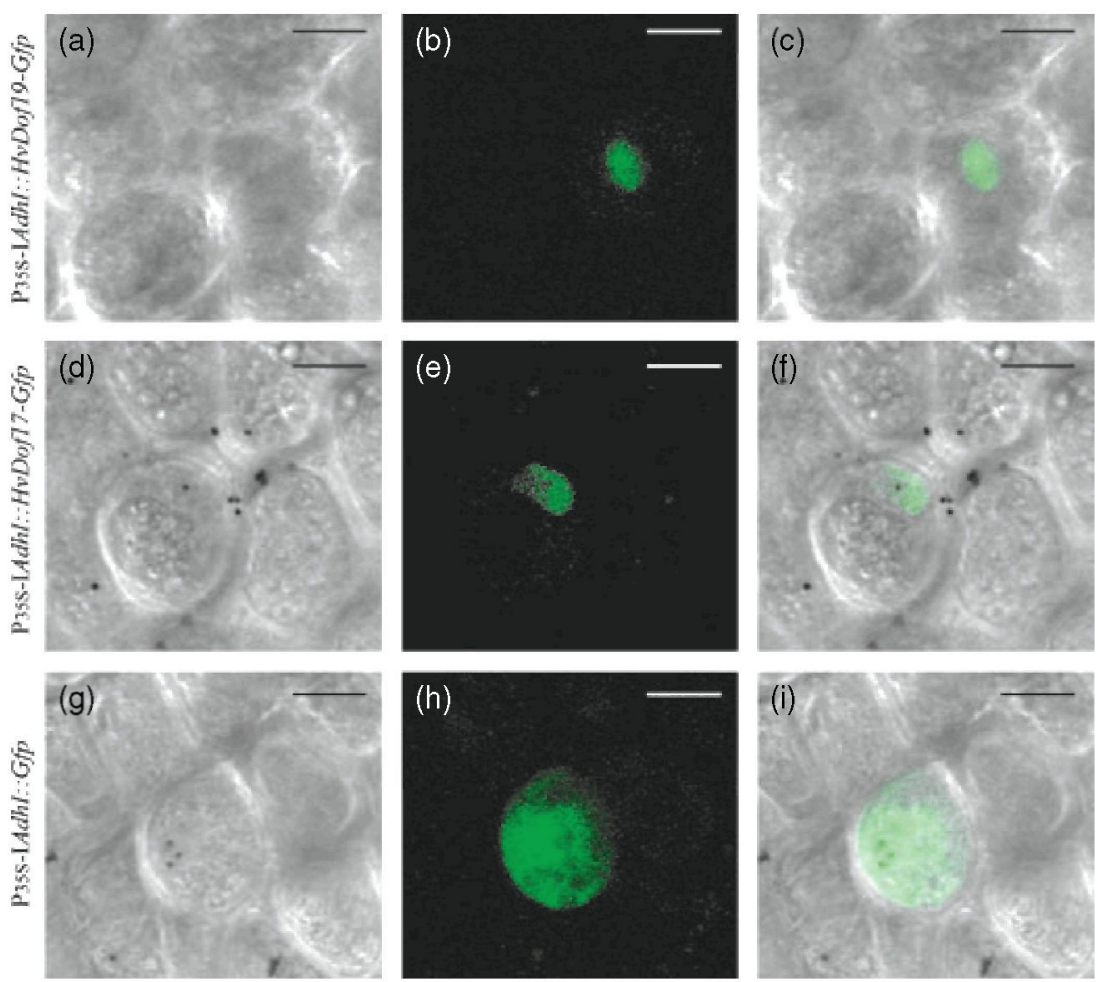

Figure 3. Sub-cellular location of HvDOF19 and HvDOF17 in aleurone cells.

Aleuronelayers cells were bombarded with the $\mathrm{P}_{35}$-IAdhl:HvDof19-Gfp construct $(\mathrm{a}, \mathrm{b}, \mathrm{c})$, the $P_{35}-I A d h l:$ HvDof77-Gfp construct $(d, e, f)$ or the $P_{35}-\mid A d h l:: G f p$ control constructs $(\mathrm{g}, \mathrm{h}, \mathrm{i})$ and incubated for $24 \mathrm{~h}$ before observation under a confocal ultraspectral microscope LEICA TCSSp2.AOBS-UV for the emission spectrum of the GFP (b, e, h) or under Nomarski $(a, d, g)$. The Nomarski and fluorescence photopgraphs were overlapped $(c, f, i)$. Scale bars: $20 \mu \mathrm{m}$. spread throughout the cell (Figure $3 g-i$ ). Nomarski pictures (Figure $3 a, d, g$ ) and the overlapping photographs (Figure $3 c, f, i)$ of these and the fluorescence images (Figure $3 b, e, h$ ) are also shown.

\section{HvDOF19 and HvDOF17 are transcriptional repressors of the GA-responsive gene Al21 in aleurone}

The expression patterns and hormonal responses of HvDof19 and HvDof17 suggested that they could be regulators of the expression of hydrolase-encoding genes in germinating aleurone cells. Thus, we addressed whether these factors could modulate the transcriptional activity of the promoter of the Al21 gene, which encodes a cathepsin B-like protease, in transient expression assays by particle bombardment of isolated layers of barley cv. Himalaya. As a reporter (Figure 4a), we used the pCBG13 construct (Cejudo et al., 1992) where the GUS gene was under the control of a 480-bp promoter fragment (from the translation start codon) of the A/21 gene that contained three pyrimidine boxes and two GARE motifs. These two cis-motifs were recognized by DOF TFs and GAMYB, respectively (IsabelLaMoneda et al., 2003; Mena et al., 2002). The effector constructs were the ORFs of HVDof17, HVDof19 and GAMyb under the control of the CaMV35S promoter, followed by the first intron of the $A d h /$ gene from maize and flanked downstream by the $3^{\prime}$ NOS terminator (Figure 4a). Isolated aleurone layers were bombarded either with the reporter alone or in combination with the effectors at a 1:1 molar ratio. The
GUS activity obtained after bombardment of the reporter without effectors was arbitrarily assigned the $100 \%$ value. When co-transfection of pCBG13 with HvDof19, HvDof17 or with both effectors was performed either in the presence or in the absence of GA, GUS expression was drastically reduced (Figure 4b). Co-transfection with the GAMyb construct as effector produced an increase in GUS activity of $500 \%$, as previously described (Gubler et al., 1995, 1999). Co-bombardment of pCBG13 with GAMyb plus HvDof17 or plus HvDof19 (at a 1:1:1 ratio) dramatically decreased GUS expression.

In addition, we assayed a shorter version of the $A / 21$ gene promoter (Cejudo et al., 1992) that only contained one DOF (D2) and two R2R3 MYB (M1 and M2) putative binding sites (Isabel-LaMoneda et al., 2003). Similar patterns of GUS activity mediated by HvDof 17 and HvDof19 transient expression were obtained (data not shown). The GUS activity measured for this shorter version of the $A / 21$ gene promoter was $70 \%$ of that measured for the $\mathrm{pCBG} 13$ construct. We can thus infer that the cis-motif D2 was sufficient for the negative regulation of the A/21 gene promoter by the HvDOF19 and HvDOF17 TFs.

Silencing of HvDof19 by dsRNAi prevents the ABA-mediated repression of the Al21 gene in aleurone

As RT-qPCR analyses indicated that HvDof19 was ABAinduced (sixfold), and that HvDOF19 was a transcriptional repressor of the $A / 21$ gene promoter in transient expression 
(a)

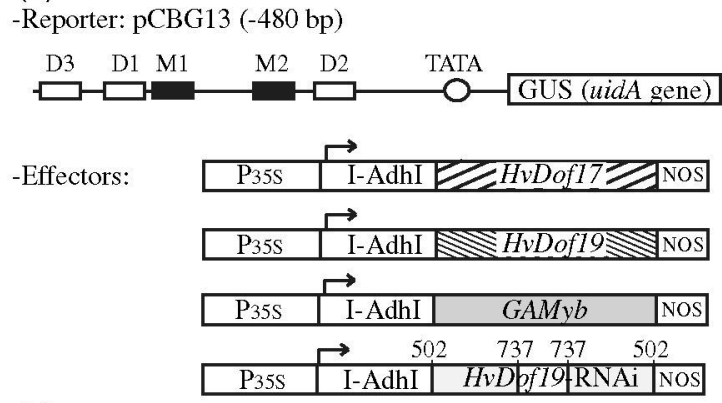

(b)

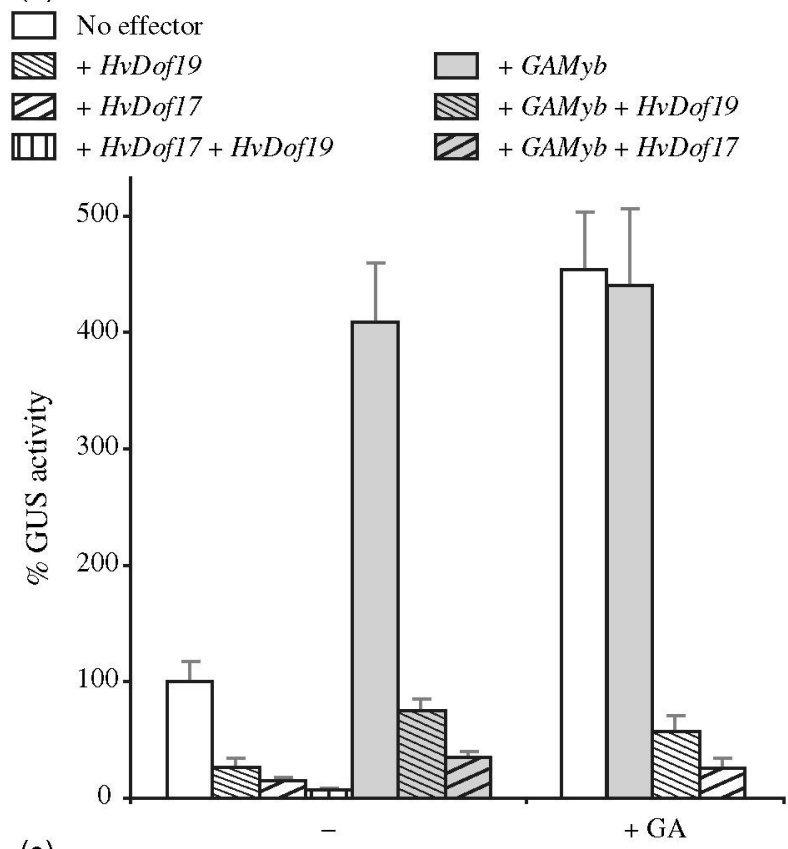

(c)

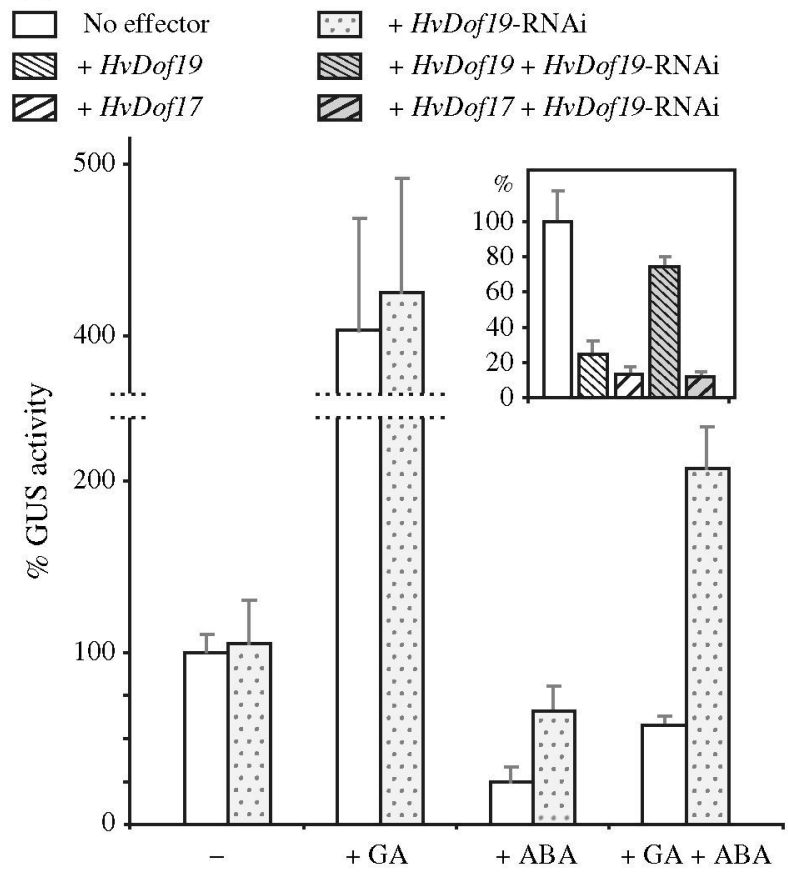

experiments, we decided to explore whether this TF could have a role in the $A B A$-mediated repression of $A / 21$ gene expression in the aleurone. To address this question we transiently expressed, in this tissue, a double-stranded RNAi (ds-RNAi) construct of the HvDof19 gene together with the pCBG13 reporter described in Figure 4(a). This construct contained a specific fragment (from 502 to 737 bp from the translation start codon) of HvDof 19 in sense and antisense orientations and split by a 110-bp DNA fragment. The response to GA and ABA of the A/21 gene promoter was studied. Isolated aleurone layers were bombarded with the reporter alone or in combination with the effector at a 1:1 molar ratio, and were incubated for $24 \mathrm{~h}$ in saline buffer, used as control, or with $1 \mu \mathrm{M} \mathrm{GA}_{3}, 10 \mu \mathrm{M}$ ABA or a mixture of both hormones. As shown in Figure 4(c), when the reporter was incubated with GA, GUS activity increased by $400 \%$ over that obtained when the reporter was used alone without hormones. And when incubated with ABA, GUS activity decreased by $75 \%$. The HvDof19-RNAi construct did not have any effect either in the control or upon GA incubation. However, co-bombardment of the reporter with the HvDof19-RNAi construct decreased the repressor effect of ABA (from 25 to $65 \%$ of GUS activity), and when incubating with $A B A+G A$, the $60 \%$ repression in the absence of the RNAi was transformed into about $200 \%$ activation. In addition, the specificity of HvDof19 RNAi construct was demonstrated in the insert of Figure 4(c). The HvDof 19 repression effect on the GUS activity driven by the Al21 promoter was reversed by the HvDof19 RNAi. However, the repression mediated by HvDof17 was not affected upon bombardment with HvDof17 + HvDof19 RNAi. These results indicated that HvDOF19 had a role mediating the ABA response of the A/21 gene promoter. No effects were found when similar experiments were performed with HvDof17-RNAi (data not shown).

Figure 4. Transient expression assays by bombardment of aleurone layers from de-embryonated barley seeds with the indicated combinations of reporter and effector constructs and subsequent incubation with hormones. (a) Schematic representation of the reporter and effector constructs. The reporter construct pCBG13 is the same as that in Cejudo et al. (1992). Blank and black boxes indicate putative cis DNA binding sites for DOF and MYBR2R3 transcription factors (TFs), respectively. The effector constructs comprised the ORFs of HvDof77, HvDof79, GAMyb and an RNAi construct for HvDof19 under the control of the CaMV35S promoter $\left(P_{35}\right)$ followed by the first intronof the maize $A d h /$ gene (I- $A d h)$. The RNAi construct contained a fragment of the HvDof79 cDNA from 502 to 737 bp from the translation initiation codon in sense and orientations separated by a stuff DNA of $110 \mathrm{bp}$.

(b) GUS activity under the control of the AI21 promoter after co-bombardment with different combinations of HVDof 17, HVDof 19 and GAMyb were analysed after being incubated in saline buffer in the absence and in the presence of gibberellins (GA).

(c) Expression of GUS activity controlled by the Al21 promoter after cobombardment with different combinations of HvDof17, HvDod19 and the RNAi construct for HvDof 19. GUS activity was analysed after incubation with $\mathrm{GA}$, abscisic acid (ABA) or GA + ABA. GUS activity was expressed as the percentage of GUS activity relative to the control without effectors. In each case, three replicates were performed and sets of five aleurones were bombarded in each experiment. Standard errors are indicated. 


\section{HVDOF19 and HVDOF17 interact with GAMYB at plant cell nuclei}

The finding that the trans-activation of the Al21 gene promoter mediated by GAMYB in aleurone layers, was reversed by HvDOF17 and HVDOF19 suggested that the two DOF proteins might interact with GAMYB in vivo. To this purpose, two different approaches were used: the Bimolecular Fluorescence Complementation (BiFC) assay to detect protein-protein interactions in plant cell nuclei, and the Yeast Two-Hybrid System (Y2HS).

For the BiFC experiments, the ORFs of HvDof19, HvDof17 and $G A M y b$ were translationally fused, in all combinations, to two non-fluorescent fragments (N-terminal GFP:GFPNH ${ }_{2-}$ or C-terminal GFP:GFPCOOH-) of the GFP, as described by Diaz et al. (2005). When interaction of two proteins fused to these two GFP fragments take place, the fluorophore is reconstituted and fluorescence is emitted. The co-bombardment of epidermal onion cells with the combinations

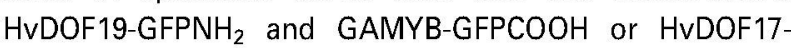
$\mathrm{GFPNH}_{2}$ and GAMYB-GFPCOOH, or in the reverse order, resulted in the GFP reconstitution after incubation for $48 \mathrm{~h}$ as observed under a confocal microscope (Figure $5 \mathrm{a}$ ). This detected green fluorescence was targeted into the onion cell nuclei. To confirm that protein-protein interaction ocurred at the cellular nucleus, DAPI staining that specifically marks DNA was used. Co-localization of the green and blue fluorescence when both photographs were overlapped demonstrated a clear spatial location (Figure 5a). In contrast, no reconstitution of the fluorescence was detected when both DOF proteins (HvDOF19 and HvDOF17) were fused to the complementary GFP fragments, or when these GFP fragments were tested alone (data not shown). These results indicated that both DOF19 and DOF17 interacted with

Figure 5. Protein-protein interaction of HVDOF19 and HVDOF17 with GAMYB in Allium cepa cell nuclei and in the yeast Saccharomyces cerevisiae twohybrid system (Y2HS).

(a) Nuclear localization of the reconstituted GFP complexes in transiently transformed onion epidermal cells. Fluorescence appeared at the nucleus after the co-bombardment with HvDOF19-GFPNH ${ }_{2}$ and GAMYB-GFPCOOH

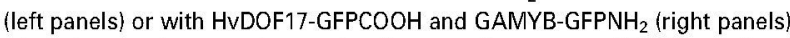
when the samples were observed under green fluorescence (GF) in a confocal ultraspectral microscope (LEICA TCS-Sp2-AOBS-UV). Observation under Nomarski bright field (NBF) and under blue fluorescence (BF) showed that reconstituted GFP co-localized with the DAPI-stained nucleus when the pictures were overlapped (O). Scale bars: $50 \mu \mathrm{m}$.

(b) Schematic representation of the reporter and effector constructs used for the $\mathrm{Y} 2 \mathrm{HS}$ assays. Gal1 UAS, Gal4 responsive element in the Gal1 promoter; Gal4BD, Gal4 DNA-binding domain; Gal4AD, Gal4 DNA activation domain GAMYB, full-length open reading frame (ORF); the $\mathrm{NH}_{2}-\mathrm{COOH}$-terminal and DOF domain regions of HvDOF19 and HvDOF17 are those indicated in Experimental procedures.

(c) The combinations of constructs 1-5 were used to transform $S$. cerevisae strain SFY526 and LacZ induction was measured to test the interaction between HvDOF19 and GAMYB

(d) The combinations 1 and 6-9 were used to test the interaction between HvDOF17 and GAMYB. GUS activity in liquid cultures was calculated from six independent replicates in two different assays. Standard errors are indicated. (a) HvDOF19-GFPCOOH::GAMYB-GFPNH 2 HvDOF17-GFPNH $2:$ GAMYB-GFPCOOH

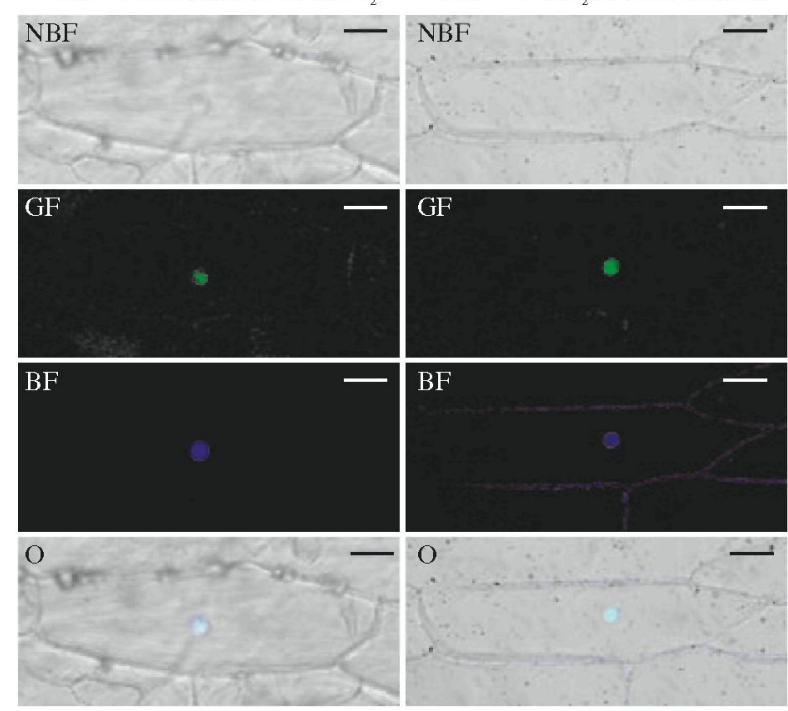

(b)

Reporter:

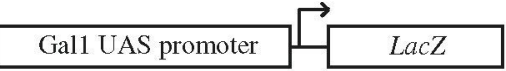

Effectors:

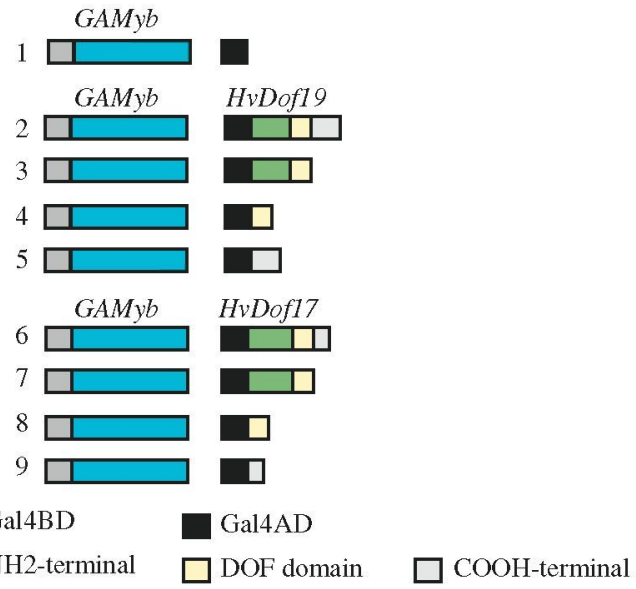

(c)

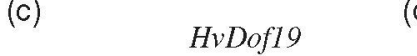

(d)
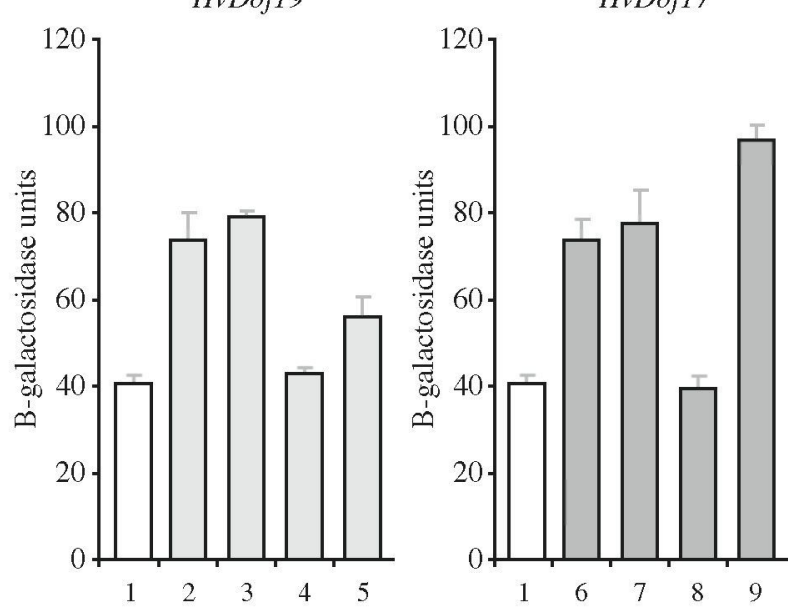
GAMYB in plant cell nuclei, but that they do not form homoor hetero-dimers between them.

To ascertain what parts of HvDOF19 and HvDOF17 proteins were responsible for the interaction with GAMYB, $\mathrm{Y} 2 \mathrm{HS}$ experiments were performed using the $\mathrm{LacZ}$ gene as reporter. As represented schematically in Figure $5(b)$, the GAMYB protein was expressed as a translational fusion to the yeast Gal4 binding domain (BD), and a series of constructs containing either the whole ORFs of HvDOF19 or HvDOF17, or three different fragments derived from them, were fused to the yeast Gal4 activation domain (AD). These constructs were used to transform Saccharomyces cerevisiae yeast cells (strain SFY526) and the corresponding LacZ reporter activity was quantified in liquid medium. When yeast cells were transformed with the Gal4BD-GAMYB fusion construct, the $\beta$-galactosidase activity values were about 40 Miller units, indicating that GAMYB functions as a transcriptional activator in yeast. Combinations of Gal4BDGAMYB with HvDOF17 or its N-terminal part almost doubled this activity, whereas the fusion of the GaL4AD to its Cterminal part reached a $\beta$-galactosidase activity of 100 units (Figure 5b,d). However, no increase above GaL4BD-GAMYB activity was detected when the 52 residues of the DNAbinding domain were fused in frame to the GaL4AD. This indicated that HvDOF17 interacted with GAMYB by both its $\mathrm{N}$ - and $\mathrm{C}$-terminal regions, but that the DOF domain did not participate in this interaction. Similar results were obtained for the HvDOF19-GAMYB interaction. However, in this case the increase in the activity that showed the interaction of GAMYB with the C-terminal region of HvDOF19 was about 1.5-fold higher than when GaL4BD-GAMYB was used alone (Figure $5 b, c$ ).

HvDOF19 binds to the three DOF motifs in the Al21 gene promoter in vitro, and HvDOF17 affects the binding affinity of GAMYB for its recognition site CAACG/C

As HvDOF17 and HvDOF19 mediated the repression of the Al21 gene promoter in transient expression experiments, and as the sequence of this promoter presents three putative DOF-binding motifs (Isabel-LaMoneda et al., 2003), we tested if both proteins were capable of binding specifically to these motifs in vitro through electrophoretic mobility shift assays (EMSA).

The HvDOF17 and HvDOF19 proteins were expressed as GST fusions in Escherichia coli, and were incubated with the three radioactively-labeled oligonucleotide probes D1, D2 and D3 derived from the Al21 gene promoter. As shown in Figure $6(\mathrm{a}), \mathrm{GST}-\mathrm{HvDOF} 19$ bound to the three probes in vitro, although the retardation band detected for the D3 probe was considerably fainter. Binding specificities were confirmed by competition titrations, up to 100X molar excess, with the corresponding non-labeled probes D1 and D2. The mutated probes $\mathrm{d} 1, \mathrm{~d} 2$ and $\mathrm{d} 3$, in which the AAAG
Figure 6. Electrophoretic mobility shift assays (EMSAs) of the recombinant GST-HvDOF17, GST-HvDOF19 and GST-GAMYB proteins with probes derived from the $A / 21$ gene promoter. (a) EMSA of the recombinant HvDOF19 protein with the ${ }^{32} \mathrm{P}$-labeled probes D1, D2 and D3 containing DOF-binding target sequences in bold, or their mutated versions, d1, d2 and d3, in lower letters. Competition experiments were performed using increasing quantities (20X, 50X and $100 \mathrm{X}$ ) of the indicated unlabeled wild-type or mutated probes.

(b) EMSA of serial dilutions of GST-GAMYB with the ${ }^{32} \mathrm{P}$-labeled probe $\mathrm{M} 1$ that contains a R2R3MYB binding site, and GST-HvDOF17, GST-HvDOF19 or GST, used as control. (a)
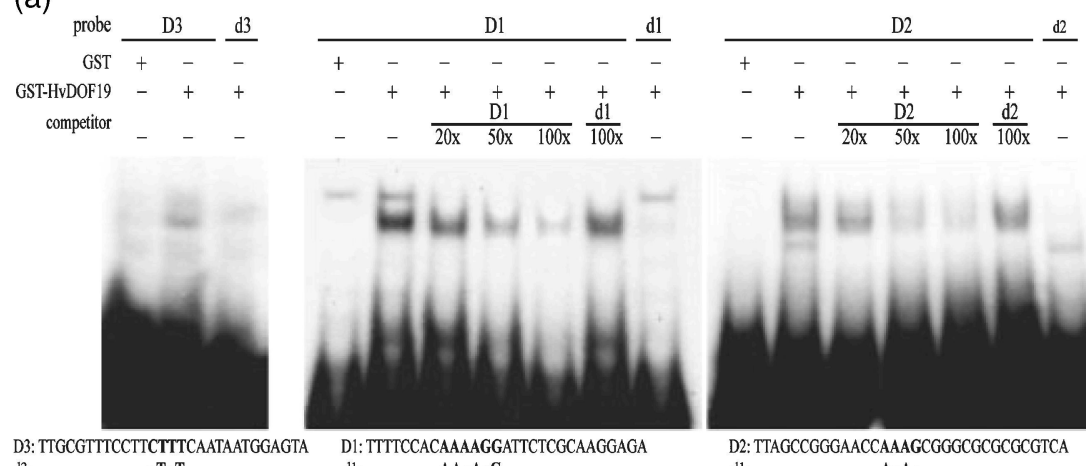

d3: -

(b)

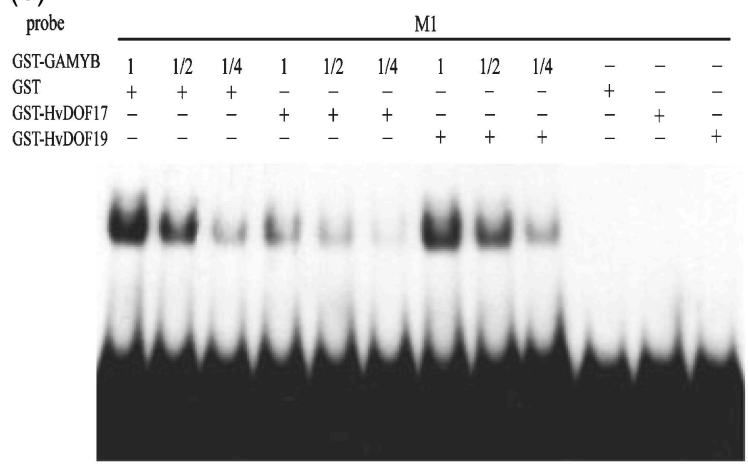

M1: TTGAAGCAACGGCAACGCATGGGCGGCGAA 
core sequence was changed to $\mathrm{AgAc}$, neither produced any shifted complex nor competed with the labeled probe, even when used at 100X molar excess. As expected, binding was not detected when the GST protein expressed in E. coli was assayed. No shifted bands were observed in similar experiments when incubation was carried out with the GSTHvDOF17 recombinant protein (data not shown).

Based on the fact that the trans-activation exerted by GAMYB in transient expression experiments in aleurone was reversed by HvDOF17 and HvDOF19, and that both DOF factors interacted in vivo with GAMYB, we decided to investigate whether these interactions might affect the binding capacity of GAMYB to its specific-target motifs in the $A / 21$ gene promoter. EMSA experiments were performed using GAMYB expressed as a GST fusion protein, the M1 probe, which included the MYBR2R3 motif located at position $-367 \mathrm{bp}$ of the A/21 promoter (Isabel-LaMoneda et al., 2003), and the recombinant GST-HvDOF17 and GSTHvDOF19 proteins. As shown in Figure 6(b), a clear decrease in the intensity of the retarded band was observed when the GAMYB protein was incubated with the HvDOF17 protein, whereas no effect was observed when the HvDOF19 protein was used in the assay.

\section{Discussion}

Seed germination begins with water imbibition, proceeds with the elongation of the radicle tip inside the seed and ends when the radicle protrudes from the seed coat (Bove et al., 2001). This process and the subsequent post-germination events are mediated by hormones, particularly $G A$ and $A B A$. GA activation and $A B A$ repression of hydrolase genes in the cereal aleurone are key processes that regulate the proper supply of nutrients from the endosperm to the embryo until the photosynthetic capability is completely acquired. In barley, GA promotes the degradation of the DELLA protein SLN1 that finally induces the expression of GAMYB, which is the principal transcriptional activator of hydrolase gene expression (Fu et al., 2002; Kaneko et al., 2004; Sun and Gubler, 2004). However, the ABA-mediated repression of these genes is a mechanism not yet well understood. This article provides novel insights into the ABA regulation of genes encoding hydrolases, and proposes a role for HvDOF19 in the ABA-dependent pathway. HvDOF17 and the previously reported BPBF (Mena et al., 2002) would also contribute to the negative regulation of hydrolase gene expression in germinating aleurone, but in an ABA-independent pathway.

Evidence of the ABA negative regulation of hydrolase gene expression put forward the existence of various mechanisms of regulation. Firstly, ABA would act through the GA-positive regulator GAMYB, as its mRNA levels decrease by the addition of ABA (Gubler et al., 1995, 2002). However, the $A B A$ repression of hydrolase gene expression is independent of the GA-signaling transduction pathway and acts downstream of the barley SLN1 protein because: (1) ABA suppresses the derepression of $\alpha$-amylase genes that occurs in the absence of GA when RNAi technology is used to inhibit the SLN1 synthesis (Zentella et al., 2002); and (2) the GA-induced degradation of SLN1 is not blocked by ABA (Gubler et al., 2002). In addition, ABA only partially counteracts the GA-induced expression of GAMYB (Gubler et al., 2002), so there must be other components/factors implicated in the $\mathrm{ABA}$ repression of hydrolase genes. The $\mathrm{ABA}$-induced protein kinase PKABA 1 inhibits the GA induction of both GAMyb and hydrolase gene expression when this hormone is externally added (Gomez-Cadenas et al., 1999, 2001b; Shen et al., 2001). However, PKABA1 has no effect on GAMYBtransactivated hydrolase gene expression, and the use of RNAi to block its synthesis does not affect the ABA-mediated repression of $\alpha$-amylase genes (Gomez-Cadenas et al., 2001b; Zentella et al., 2002). This suggests that an alternative pathway independent of this kinase must exist. Our results indicate that HvDOF19, the mRNA levels of which are highly induced by $A B A$ in aleurone layers (sixfold, Figure 2b), represses the expression of the $A / 21$ gene promoter both in the presence and in the absence of GA in transient expression experiments (Figure 4b). In addition, when we simultaneously use HvDOF19 and GAMYB as effectors, we observe that HvDOF19 suppresses the GAMYB-induced expression of the GUS gene under the control of the $A 121$ gene promoter. As the mRNA levels of HvDof19 are still induced in aleurones incubated with ABA plus GA, the DOF19 transcription factor could mediate the $A B A$ repression of hydrolase genes both in the presence of GA and in its absence. This was further supported by RNAi experiments silencing the HvDof19 gene transcripts. As shown in Figure 4(c), the ABA-mediated repression of the $A / 21$ gene decreased by at least $60 \%$ when HvDof19 was silenced and both ABA alone and the mixture of $A B A$ and $G A$ were added to the aleurone incubation medium. HvDOF17 also represses the expression of the Al21 gene promoter in transient expression experiments (Figure 4b). However, the expression of the HvDof17gene is not affected by incubation with ABA (Figure 2b), and RNAi experiments silencing HvDof17 do not support a role for this gene in the ABA-mediated regulation of hydrolase genes (data not shown). Therefore, HvDOF17 would negatively regulate Al21 gene expression independently of the ABA pathway.

Other genes from cereals participating in the ABA-mediated repression of hydrolase genes in the aleurone are OsWrky51 and OsWrky71 from rice. Both genes are induced by $A B A$, and their corresponding proteins repress the $G A$ induced expression of hydrolase genes through binding to the 5'-TGAC-3' sequence within the O2S box (Xie et al., 2006; Zhang et al., 2004). Although this sequence is present in lowpl $\alpha$-amylase gene promoters, it seems to be absent in the high-pl $\alpha$-amylase gene promoters (Lanahan et al., 1992). In addition, the developmental regulator VIVIPAROUS (VP1) 
from maize, which is required for $A B A$-induced gene expression during seed maturation (Rock and Quatrano, 1995 and references cited therein), has been described to specifically repress the induction of $\alpha$-amylase genes by GA in aleurone of germinating barley seeds (Hoecker et al., 1995). However, no direct interaction with hydrolase gene promoters has been reported.

Our results also show that HvDOF19 specifically binds to the pyrimidine boxes in the promoter of the A/21 gene. On the contrary, HvDOF17 does not seem to bind directly to any of these putative DOF-binding motifs in vitro (EMSA). However, it is able to repress the expression of the Al21 gene, probably by decreasing the binding affinity of the GAMYB protein to its target site in this promoter (Figure 6b). In addition, protein-protein interactions between GAMYB and HvDOF19 and HvDOF17 in plant nuclei have been observed, pointing to their participation in a combinatorial regulatory complex with GAMYB and other DOF TFs, such as BPBF and SAD (Figure 7).

Over the past few years information regarding variation of ABA and GA content in germinating barley seeds has been reported (Jacobsen et al., 2002; Millar et al., 2006). After imbibition, ABA mainly decreases in non-dormant grains, and once its levels are reduced (which seems to occur around 18 hai), biologically active $\mathrm{GA}$ begins to accumulate. In Arabidopsis, the GA requirements to promote germination seem to be determined by embryonic ABA (Ali-Rachedi et al., 2004; Debeaujon and Koornneef, 2000), which would be de novo synthesized to maintain dormancy in imbibed seeds (Koornneef et al., 2002). Our results show that in aleurone layers HvDof19 mRNA levels peak at 16 hai, just before radicle emergence (18 hai), only to fall drastically thereafter. According to this GA/ABA profile, HvDof19 mRNAs could be induced by ABA before it is catabolized, and before the GA synthesized by the embryo diffuses to the aleurone. Later on, when these cells change to a high $\mathrm{GA}$ / ABA ratio, a decrease in the transcript levels of the HvDof19 gene would take place. Interestingly, HvDof17, HvDof19 and the barley Pbf present similar expression patterns, although neither HvDof17 nor Pbf are induced by ABA. GA would contribute to the decrease in the HvDof17 and HvDof19 mRNA levels, whereas for the HvDof24-Pbf gene, which is GA induced (Figure $2 \mathrm{~b}$ and Mena et al., 2002), the 50\% detected decrease at 24 hai might be motivated by the disappearance of some other unknown factor(s) that would have caused its induction up to fourfold at 16 hai.

We propose a model of negative regulation of hydrolase gene expression mediated by DOF TFs in aleurone layers. In this model, when the ABA level is still high, as occurs in the early germinative phase, HvDOF19 would mediate the ABA repression of hydrolase genes. HvDOF17 and BPBF would help in this process independently of the ABA pathway (Figure $7 b$ ). In the post-germination, when the ABA level has decreased and the GA diffusion from the scutellum has been (a)
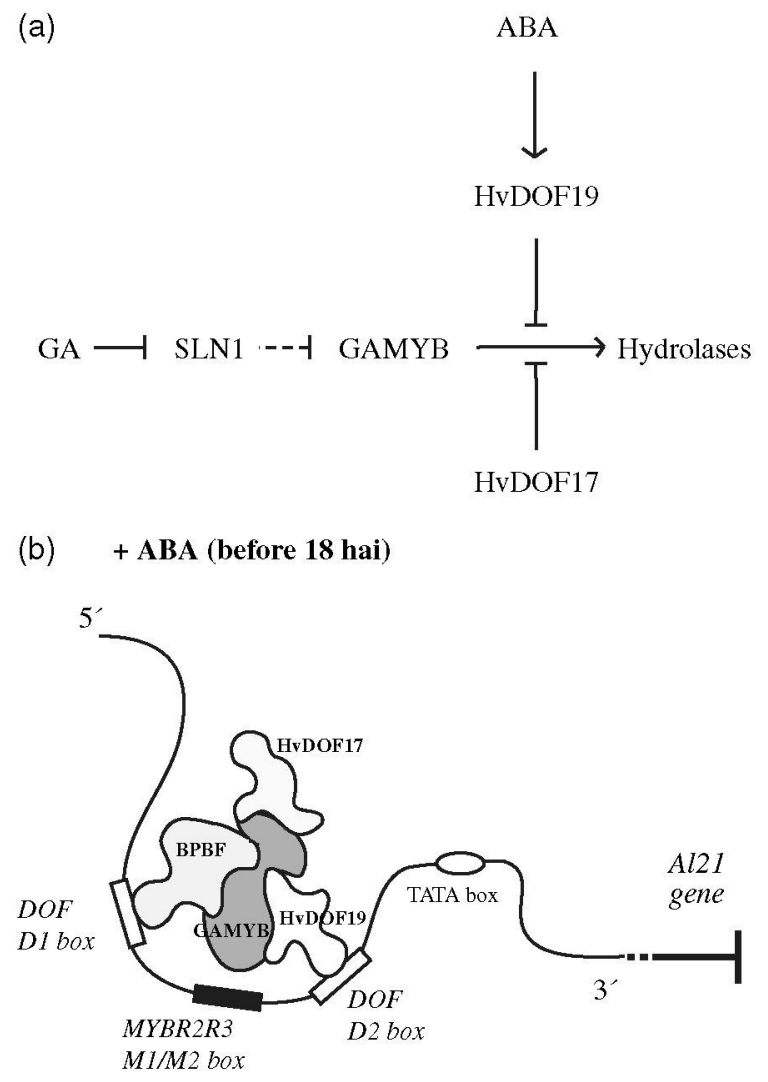

$\begin{array}{ll}\text { (c) } & + \text { GA (after } 18 \text { hai) } \\ & - \text { ABA } \\ 5 & \end{array}$

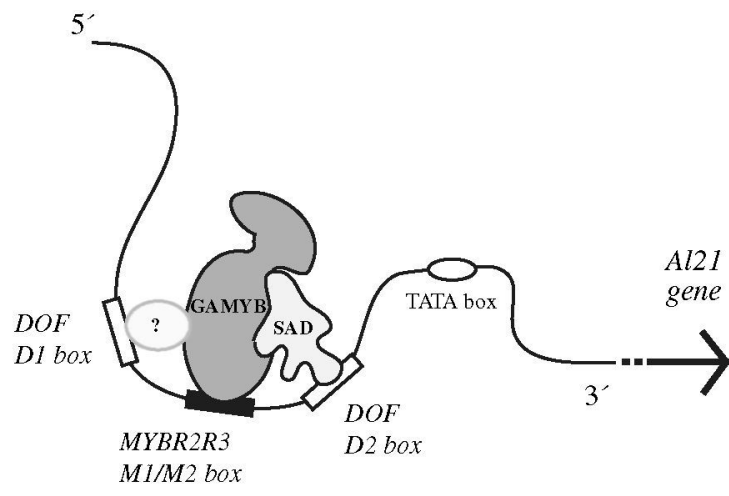

Figure 7. Proposed model of transcriptional regulation of abscisic acid repressed/gibberellins induced expression of hydrolase genes in barley aleurone layers mediated by DOF TFs and GAMYB.

(a) The diagram summarizes the proposed role of HvDOF17 and HvDOF19 in the suppression of hydrolytic enzymes expression in barley aleurone.

(b) In an early phase of germination (before 18 hai) when the ABA level is high, negative regulation would be exerted by HvDOF17, HvDOF19 and BPBF on the promoter of the $A / 21$ gene.

(c) In a later phase of germination (post-germination, after 18 hai), when the GA/ABA ratio is high, GAMYB and other transcriptional activators would trigger the GA-mediated transcriptional activation of the Al21 gene.

Transcription factors (TFs) are indicated as jigsaw puzzle pieces (HvDOF17, HvDOF19, BPBF, GAMYB and SAD) and DNA target sequences for each class of TF are boxed. 
produced, the quantity of these TFs would be insignificant compared with that of the positive effectors such as GAMYB, SAD and other DOF and R1MYB activators (Rubio-Somoza et al., 2006a,b). Therefore, transcription of aleurone hydrolase genes would be carried out (Figure $7 \mathrm{c}$ ).

\section{Experimental procedures}

\section{Cloning of HvDof19 and HvDof17}

The ORFs of HvDof19 and HvDof17 (Moreno-Risueno et al., 2007) were amplified from first-strand cDNA of germinating barley seeds $\mathrm{cv}$. Bomi by PCR using the primers 5'-ATGCTCTCCCACGTC-3' and 5'-TCAGAACCGGGGGTC-3' for HvDof17 and 5'-ATGGTGTCTCACTCTC-3' and 5'-TCATGCGTATTGGTTG-3' for HvDof 19. The resultant amplified fragments were cloned in pGEM T-easy (Promega, http:/(www.promega.com) and further used for their molecular and functional characterization.

\section{$R T-q P C R$ analyses}

RT-qPCR analyses were performed to study variation in the rate of transcription of the genes HvDof2, HvDof8, HvDof17, HvDof19, HvDof23-Sad, HvDof24-Pbf (encoding DOF factors), GAMyb (encoding a R2R3MYB factor) and CatB (encoding a cathepsin B-like protease) in aleurone cells and in embryos upon germination, as well as in isolated aleurone layers in response to GA and $A B A$ treatments. Seeds of barley cv. Himalaya were sterilized in $1.7 \%$ $\mathrm{NaOCl}$ for $10 \mathrm{~min}$, washed in distilled water and incubated on water imbibed filter paper at $22^{\circ} \mathrm{C}$ in the dark. Aleurone layers and embryos were collected at different incubation times. For hormone treatments, seeds of barley cv. Himalaya were de-embryonated, sterilized and incubated for $48 \mathrm{~h}$ in the dark at $22^{\circ} \mathrm{C}$ on filter paper soaked with calcium succinate buffer $(20 \mathrm{~mm}$ Na-succinate, $20 \mathrm{~mm}$ $\mathrm{CaCl}_{2}, \mathrm{pH}$ 5.2). Then, aleurone layers were isolated under a dissecting microscope and incubated for $20 \mathrm{~h}$ in the same buffer containing $1 \mu \mathrm{MGA}, 10 \mu \mathrm{M} A B A$ or a mixture of both hormones, or with no hormones as controls. Total RNA was purified from frozen tissues by phenol/chloroform extraction, followed by precipitation with $3 \mathrm{M} \mathrm{LiCl}$ (Lagrimini et al., 1987) and digestion with DNase (Dnase I, RNase-free; Roche Diagnostics, http://www.roche.com). These samples $(20-50 \mu \mathrm{g})$ were used to isolate mRNA (QuickPrep ${ }^{\top \mathrm{M}}$ Micro mRNA Purification Kit; GE Healthcare, http://www. gehealthcare.com), and duplicate first-strand cDNA samples were synthesized in the presence of a $17 \times T$ oligonucleotide according to the manufacturer's instructions (first-strand cDNA Synthesis Kit; GE Healthcare). RT-qPCR analyses were performed for each of the duplicate samples by means of a 7300 Real Time PCR System (Applied Biosystems, http://www.appliedbiosystems.com) using an SYBR-green detection system for HvDof2, HvDof8, HvDof23-Sad, HvDof24-Pbf, GAMyb and CatB genes, and for HvDof17 and HvDof19 genes only in the analysis shown in Figure 1(a), and a TaqMan MGB probe detection system for HvDof17 and HvDof19 in the rest of the analyses. Quantification was standardized to the barley Actin 2 mRNA levels, and when indicated were referred to the control value that was arbitrarily set to 1 . The primers used for PCR amplification were as follows: barley Actin2 (SYBR-green detection system: forward, 5'-TCCAGCTATCGTCCACAGGAA-3'; reverse, 5'TGTCCAACAAAACCACCACCT-3; TaqMan MGB probe detection system: forward, 5'-TCCACCGGAGAGGAAGTACAGT-3'; reverse, 5'AATGTGCTCAGAGATGCAAGGA-3'), HvDof17 (SYBR-green detection system: forward, 5'-TGTTCCTTCCCCTGGTTTCTT-3'; reverse,
5'-TTGATGCTACTACCGCGCAAC-3'; TaqMan MGB probe detection system: forward, 5'-ACAGACTCGGGCTTGGAAGA-3'; reverse, 5'CCGTCGCGTCACGATTC-3'), HVDof19 (SYBR-green detection system: forward, 5'-TGGCTCGTTCATCAGTACCCA-3'; reverse, 5' CGGCAAGAAATGCAAGTTGG-3; TaqMan MGB probe detection system:forward, 5'-CTCGGCCACGGAGAGATG-3'; reverse, 5'-TT GGGCTGGGTACTGATGAAC-3'), HvDof2 (forward, 5'-ATATGC ATGGCTCATGCGATC-3'; reverse, 5'-CCAATGCCACCACCATCTT ATT-3'), HvDof8 (forward, 5'-CACACCCACAAAGCCACTGATA-3'; reverse, 5'-TGGACTGGAAGTCTTGCATGG-3'), HvDof23-Sad (forward, 5'-TGCTCTAGCCCGAGGACATAAA-3'; reverse, 5'-GGAATG TCTTGTGGATGACCGT-3'), HvDof24-Pbf (forward, 5'-GGAAATG GAATAGGTGGCACAA-3'; reverse, 5'-TACCAACATGTGGCTGCCACT-3'), GAMyb (forward, 5'-TGTGATACCACGGTGGTTAGCC-3'; reverse, 5'-GGATCCAGTGAGAGAATTGCCA-3') and CatB (forward, 5'-TCGATGAAGAACATCGCCG-3'; reverse, 5'-TGCCCGCTTAATTT GACAGG- $3^{\prime}$ ). The sequences of the TaqMan MGB probes were: Actin2 (5'-TCTGGATCGGAGGGTC-3'), HvDof17 (5'-AGAGTTT GGTTGACCCGC-3') and HvDof19 (5'-AGCAGTGCTGGTGGC-3').

\section{In situ hybridization}

Seeds of barley cv. Bomi were sterilized in $1.7 \% \mathrm{NaOCl}$ for $10 \mathrm{~min}$, washed in distilled water and germinated at $22^{\circ} \mathrm{C}$ in the dark for $16 \mathrm{~h}$. Aleurone layers and embryos were collected, fixed in $4 \%$ formaldehyde in PBS, dehydrated, embedded in paraffin and sectioned to $10 \mu \mathrm{m}$. After de-waxing in Histoclear, the samples were rehydrated, treated with cellulase $2 \%$ in PBS, with $0.25 \%$ acetic anhydride in $0.1 \mathrm{M}$ triethanolamine and dehydrated. Hybridization was performed overnight at $50^{\circ} \mathrm{C}$ in hybridization solution $\left(100 \mu \mathrm{g} \mathrm{ml}^{-1}\right.$ tRNA, $6 \times$ SSC, $3 \%$ SDS and $50 \%$ formamide) plus approximately $100 \mathrm{ng} \mathrm{\mu l}^{-1}$ of the antisense or sense DIG-labelled RNA probes corresponding to HvDof17 (from 670 to 984 bp from the ATG initiation codon) and HvDof19 (from 401 to $770 \mathrm{bp}$ from the ATG initiation codon). Washes of $2 \times \mathrm{SSC}$ and $0.2 \times \mathrm{SSC}$ for $15 \mathrm{~min}$ at $50^{\circ} \mathrm{C}$ were then carried out twice. Antibody incubation and colour detection were performed according to the manufacturer's instructions (Roche Diagnostics).

\section{Subcellular localization in aleurone cells}

Translational fusions of the ORFs of the HvDof17 and HvDof19 genes to the GFP reporter gene were made by cloning both ORFs into the BamHI restriction site of a pBlueScript vector (Stratagene, http:/(www.stratagene.com) in which the GFP gene had been previously cloned into the $B a m H I-E c o R I$ sites. This plasmid already contained the CaMV35S promoter followed by the first intron of the maize $\mathrm{Adhl}$ gene and the $3^{\prime}$ NOS terminator. The generated con-

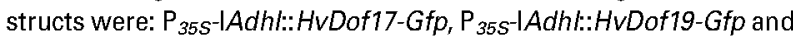
$\mathrm{P}_{35 s^{-}} \mid A d h l: G f p$ as a control. Preparation of aleurone layers, gold particle coating and bombardment with a biolistic Helium gun device (DuPont PDS-1000; BioRad, http://www.biorad.com) were performed as described in (Rubio-Somoza et al., 2006b). After $24 \mathrm{~h}$ of incubation at $22^{\circ} \mathrm{C}$ in the dark, aleurone layers were observed and photographed under a confocal ultraspectral microscope LEICA TCS-Sp2-AOBS-UV (Leica, http://www.leica.com) using the appropriated filters.

\section{Transient expression assays in aleurone layers}

The reporter constructs $\mathrm{pCBG} 13$ and pCBG13.1 containing two fragments of the wheat cathepsin B-like promoter of the Al21 gene 
were the same as those used in Cejudo et al. (1992). The effector constructs were made by cloning the whole ORFs of HvDof17 and HvDof19, or a fragment of the HvDof19 gene (from 502 to $737 \mathrm{bp}$ from the translation start codon), in sense and antisense, and separated by a 110-bp length DNA using an $\mathrm{Ncol}$ and an $\mathrm{Xbal}$ site, into the BamHI-EcoRI restriction sites of the pBlueScript vector (Stratagene) under the control of the CaMV35S promoter followed by the first intron of the maize $A d h /$ gene and the $3^{\prime}$ NOS terminator. The effector construct carrying the ORF of GAMYb and preparation of aleurone layers, gold particle coating and bombardment with a biolistic Helium gun device (DuPont PDS-1000; BioRad) were performed as described previously (Diaz et al., 2002; Isabel-LaMoneda et al., 2003). GUS expression was determined histochemically following the method of Jefferson (1987). GUS activity was calculated as the mean value of blue spots per aleurone in each assay and expressed as a percentage, considering $100 \%$ as the value obtained with the reporter constructs without effectors. The histochemical data were directly correlated with the GUS expression quantified by chemiluminescence (GUS light Kit; Tropix, Bedford, MA, USA) per milligram protein with a correlation coefficient of 0.98 (data not shown).

\section{Bimolecular fluorescence complementation}

$\mathrm{HvDof} 19$ and $\mathrm{HvDof17}$ were fused in-frame with both the $\mathrm{NH}_{2}$ - and $\mathrm{COOH}$-terminal encoding fragments of Gfp, by cloning both ORFs into the $B a m H I$ restriction site of the psmRS-GFP plasmids carrying the corresponding GFP fragments. The constructs generated were 35S::HvDOF19-GFP NH $2,35 S:: H v D O F 19-G F P ~ C O O H, 35 S:: H v D O F 17-$ GFP NH${ }_{2}, 35 S:$ HvDOF17-GFP $\mathrm{COOH}$. The constructs carrying $G A$ $M y b$ were the same as those in Diaz et al. (2005). Inner epidermal layers of fresh local purchased onions (Allium cepa) were prepared and co-bombarded as previously described (Diaz et al., 2005). The fluorescence emission was observed after $48 \mathrm{~h}$ of incubation at $22^{\circ} \mathrm{C}$ in the dark under a confocal ultraspectral microscope LEICA TCSSp2-AOBS-UV (Leica). As a control of nuclei localization, tissue samples were stained with DAPI (Serva, http://www.serva.de).

\section{Yeast transformation and LacZ assays}

Plasmids pGBT9 and pGAD424 (Clontech, http://www. clontech.com) were used to generate translational fusions. The complete ORF of GAMyb fused to the Gal4 DNA binding domain in the pGBT9 vector is the same as that in Diaz et al. (2002). The whole $H v D o f 19$ and HVDof17 ORFs and derived fragments from both genes were cloned into the EcoRI-Sall restriction sites of the PGAD424 plasmid. The derived fragments from HvDof17 and HvDof19 were: (1) the $\mathrm{NH}_{2}$-terminal region of 1-396 nt and 1-462 nt from the ATG initiation codon of HvDof19 and HvDof17, respectively; (2) the Dof domain regions of 231-396 nt and 297-462 nt of HvDof19 and $H v$ Dof17, respectively; (3) the combination of $\mathrm{N}$-terminal region plus Dof domain, and (4) the $\mathrm{COOH}$-terminal regions of 396-648 nt and 462-609 nt from HvDof19 and HvDof17, respectively. Yeast transformation was performed by the polyethylene glycol method and quantification of $\beta$-galactosidase production ( $L a c Z$ ) for the different transformants was calculated using Miller's formulas as described by Ausubel et al. (1990).

\section{Electrophoretic mobility shift assays}

The HvDOF19, HvDOF17 and GAMYB proteins were expressed in E. coli BL21-CodonPlus(DE3)-RP strain (Stratagene) by cloning their
ORFs into the BamHI restriction site of the pGEX-2T vector (GE Healthcare) as translational fusions to GST. Recombinant proteins were induced with $0.1 \mathrm{~mm}$ isopropyl- $\beta$-D-thio-galacto-pyranoside (IPTG) for $2 \mathrm{~h}$ and bacterial extracts were obtained by sonication in $8 \mathrm{~mm}$ DTT in PBS. Cells carrying the pGEX-2T vector with no insert were identically processed as negative controls. The end-labeling of the probes containing the putative consensus DOF (D1, D2 and D3) or GAMYB ( $M 1$ and $M 2$ ) DNA binding sites from the Al21 gene promoter and mutated versions ( $\mathrm{d} 1, \mathrm{~d} 2$ and $\mathrm{d} 3$ ), and DNA-protein binding reactions were performed as described by Isabel-LaMoneda et al. (2003).

\section{Acknowledgements}

We are grateful to $\operatorname{Dr}$ A. Graner and Dr N. Stein (Institut fur Pflanzengenetik und Kulturpflanzenforschung, IPK) for providing the EST clone (HI14B21) for HvDof17. Financial support from the Ministerio de Educación y Ciencia, Spain (projects no. BMC 2003-06 345 and BMC 2006-07 258) and from Universidad Politécnica de Madrid (project no. R05/ 10793) are acknowledged. MAM-R is the recipient of a predoctoral fellowship from the Comunidad de Madrid (Spain). The valuable technical assistance of Mar Gonzalez is also acknowledged.

\section{References}

Ali-Rachedi, S., Bouinot, D., Wagner, M.H., Bonnet, M., Sotta, B., Grappin, P. and Jullien, M. (2004) Changes in endogenous abscisic acid levels during dormancy release and maintenance of mature seeds: studies with the Cape Verde Islands ecotype, the dormant model of Arabidopsis thaliana. Planta, 219, 479-488.

Allan, A.C., Fricker, M.D., Wardm, J.L., Beale, M.H. and Trewavas, A.J. (1994) Two transduction pathways mediate rapid effects of abscisic acid in Commelina guard cells. Plant Cell, 6, 13191328.

Aoki, N., Scofield, G.N., Wang, X.D., Offler, C.E., Patrick, J.W. and Furbank, R.T. (2006) The pathway of sugar transport in germinating wheat seeds. Plant Physiol. 141, 1255-1263.

Arenas-Huertero, F., Arroyo, A., Zhou, L., Sheen, J. and Leon, P. (2000) Analysis of Arabidopsis glucose insensitive mutants, gin5 and gin6, reveals a central role of the plant hormone $A B A$ in the regulation of plant vegetative development by sugar. Genes Dev. 14, 2085-2096.

Ausubel, F.M., Brent, R., Dingston, R.E., Moore, D.D., Seidman, J.G., Smith, J.A. and Struhl, K. (1990) Current Protocols in Molecular Biology. New York: John Wiley \& Sons.

Bove, J., Jullien, M. and Grappin, P. (2001) Functional genomics in the study of seed germination. Genome Biol. 3, 1002.

Brocard-Gifford, I.M., Lynch, T.J. and Finkelstein, R.R. (2003) Regulatory networks in seeds integrating developmental, abscisic acid, sugar and light signaling. Plant Physiol. 131, 7892.

Cejudo, F.J., Ghose, T.K., Stabel, P. and Baulcombe, D.C. (1992) Analysis of the gibberellin-responsive promoter of a cathepsin Blike gene from wheat. Plant Mol. Biol. 20, 849-856.

Debeaujon, I. and Koornneef, M. (2000) Gibberellin requirement for Arabidopsis seed germination is determined both by testa characteristics and embryonic abscisic acid. Plant Physiol. 122, 415424.

Diaz, I., Vicente-Carbajosa, J., Abraham, Z., Martinez, M., Isabel-La Moneda, I. and Carbonero, P. (2002) The GAMYB protein from barley interacts with the DOF transcription factor BPBF and activates endosperm-specific genes during seed development. Plant J. 29, 453-464. 
Diaz, I., Martinez, M., Isabel-LaMoneda, I., Rubio-Somoza, I. and Carbonero, P. (2005) The DOF protein, SAD, interacts with GAMYB in plant nuclei and activates transcription of endospermspecific genes during barley seed development. Plant J. 42, 652-662.

Eastmond, P.J. and Russell, L.J. (2005) Hormonal regulation of gluconeogenesis in cereal aleurones is strongly cultivardependent and gibberellin action involves SLENDER but not GAMYB. Plant J. 44, 483-493.

Finkelstein, R.R., Gampala, S.S. and Rock, C.D. (2002) Abscisic acid signaling in seeds and seedlings. Plant Cell, 14, S15-S45.

Fu, X., Richards, D.E., Ait-Ali, T., Hynes, L.W., Ougham, H., Peng, J. and Harberd, N.P. (2002) Gibberellin-mediated proteasomedependent degradation of the barley DELLA protein SLN1 repressor. Plant Cell, 14, 3191-3200.

Gilroy, S. and Jones, R.L. (1994) Perception of gibberellin and abscisic acid at the external face of the plasma membrane of barley (Hordeum vulgare L.) aleurone protoplasts. Plant Physiol. 104, $1185-1192$.

Gomez-Cadenas, A., Verhey, S.D., Holappa, L.D., Shen, O., Ho, T.H. and Walker-Simmons, M.K. (1999) An abscisic acid-induced protein kinase, PKABA1, mediates abscisic acid-suppressed gene expression in barley aleurone layers. Proc. Natl Acad. Sci. USA, 16, 1767-1772.

Gomez-Cadenas, A., Zentella, R., Sutliff, T.D. and Ho, T.-H.D. (2001a) Involvement of multiple cis-elements in the regulation of GA responsive promoters: definition of a new cis-element in the Amy $32 b$ gene promoter of barley (Hordeum vulgare). Physiol. Plant. 112, 211-216.

Gomez-Cadenas, A., Zentella, R., Walker-Simmons, M.K. and Ho, T.H. (2001b) Gibberellin/abscisic acid antagonism in barley aleurone cells: site of action of the protein kinase PKABA1 in relation to gibberellin signaling molecules. Plant Cell, 13, 667-679.

Gubler, F., Kalla, R., Roberts, J.K. and Jacobsen, J.V. (1995) Gibberellin-regulated expression of a myb gene in barley aleurone cells: evidence for Myb transactivation of a high-pl alpha-amylase gene promoter. Plant Cell, 7, 1879-1891.

Gubler, F., Raventos, D., Keys, M., Watts, R., Mundy, J. and Jacobsen, J.V. (1999) Target genes and regulatory domains of the GAMYB transcriptional activator in cereal aleurone. Plant J. 17, 19.

Gubler, F., Chandler, P.M., White, R.G., Llewellyn, D.J. and Jacobsen, J.V. (2002) Gibberellin signaling in barley aleurone cells. Control of SLN1 and GAMYB expression. Plant Physiol. 129, 191-200.

Gubler, F., Millar, A.A. and Jacobsen, J.V. (2005) Dormancy release, ABA and pre-harvest sprouting. Curr. Opin. Plant Biol. 8, 183-187.

Hartweck, L.M. and Olszewski, N.E. (2006) Rice GIBBERELLIN INSENSITIVE DWARF1 is a gibberellin receptor that illuminates and raises questions about GA signaling. Plant Cell, 18, 278-282.

Hoecker, U., Vasil, I.K. and McCarty, D.R. (1995) Integrated control of seed maturation and germination programs by activator and repressor functions of Viviparous-1 of maize. Genes Dev. 15 2459-2469.

Isabel-LaMoneda, I., Diaz, I., Martinez, M., Mena, M. and Carbonero, P. (2003) SAD: a new DOF protein from barley that activates transcription of a cathepsin B-like thiol protease gene in the aleurone of germinating seeds. Plant J. 33, 329-340.

Jacobsen, J.V., Gubler, F. and Chandler, P.M. (1995) Gibberellin action in germinated cereal grains. In Plant Hormones (Davies, P.J., ed.). The Netherlands: Kluwer Academic Publishers, pp. 246271.

Jacobsen, J.V., Pearce, D.W., Poole, A.T., Pharis, R.P. and Mander, L.N. (2002) Abscisic acid, phaseic acid and gibberellin contents associated with dormancy and germination in barley. Physiol. Plant. 115, 428-441.

Jefferson, R.A. (1987) Assaying chimeric genes in plants: the GUS gene fusion system. Plant Mol. Biol. Rep. 5, 387-405.

Kaneko, M., Itoh, H., Ueguchi-Tanaka, M., Ashikari, M. and Matsuoka, M. (2002) The alpha-amylase induction in endosperm during rice seed germination is caused by gibberellin synthesized in epithelium. Plant Physiol. 128, 1264-1270.

Kaneko, M., Inukai, Y., Ueguchi-Tanaka, M. et al. (2004) Loss-offunction mutations of the rice GAMYB gene impair alpha-amylase expression in aleurone and flower development. Plant Cell, 16, 33-44.

Koornneef, M., Bentsink, L. and Hilhorst, H. (2002) Seed dormancy and germination. Curr. Opin. Plant Biol. 5, 33-36.

Lagrimini, L.M., Burkhart, W., Moyer, M. and Rothstein, S (1987) Molecular cloning of molecular DNA encoding the lignin forming perosidases from tobacco: molecular analysis and tissue specific expression. Proc. Natl Acad. Sci. USA, 84, 7542-7546.

Lanahan, M.B., Ho, T.H., Rogers, S.W. and Rogers, J.C. (1992) A gibberellin response complex in cereal alpha-amylase gene promoters. Plant Cell, 4, 203-211.

Lopez-Molina, L., Mongrand, S. and Chua, N.H. (2001) A postgermination developmental arrest checkpoint is mediated by abscisic acid and requires the $A B I 5$ transcription factor in Arabidopsis. Proc. Natl Acad. Sci. USA, 98, 47824787.

Lopez-Molina, L., Mongrand, S., McLachlin, D.T., Chait, B.T. and Chua, N.H. (2002) ABI5 acts downstream of $A B \mid 3$ to execute an ABA-dependent growth arrest during germination. Plant J. 32 317-328.

Lovegrove, A. and Hooley, R. (2000) Gibberellin and abscisic acid signalling in aleurone. Trends Plant Sci. 5, 102-110.

Martinez, M., Rubio-Somoza, I., Carbonero, P. and Diaz, I. (2003) A cathepsin B-like cysteine protease gene from Hordeum vulgare (gene $C a t B$ ) induced by GA in aleurone cells is under circadian control in leaves. J. Exp. Bot. 54, 951-959.

McCubbin, A.G., Ritchie, S.M., Swanson, S. and Gilroy, S. (2004) The calcium-dependent protein kinase HVCDPK1 mediates the gibberellic acid response of the barley aleurone through regulation of vacuolar function. Plant J. 39, 206-218.

Mena, M., Cejudo, F.J., Isabel-LaMoneda, I. and Carbonero, P. (2002) A role for the DOF transcription factor BPBF in the regulation of gibberellin-responsive genes in barley aleurone. Plant Physiol. 130, 111-119.

Millar, A.A., Jacobsen, J.V., Ross, J.J., Helliwell, C.A., Poole, A.T., Scofield, G., Reid, J.B. and Gubler, F. (2006) Seed dormancy and ABA metabolism in Arabidopsis and barley: the role of ABA 8' hydroxylase. Plant J. 45, 942-954.

Moreno-Risueno, M.A., Martinez, M., Vicente-Carbajosa, J. and Carbonero, P. (2007) The family of DOF transcription factors: from green unicellular algae to vascular plants. Mol. Genet. Genomics 277, 379-390.

Razem, F.A., Luo, M., Liu, J.H., Abrams, S.R. and Hill, R.D. (2004) Purification and characterization of a barley aleurone abscisic acid-binding protein. J. Biol. Chem. 279, 9922-9929.

Razem, F.A., El-Kereamy, A., Abrams, S.R. and Hill, R.D. (2006) The RNA-binding protein FCA is an abscisic acid receptor. Nature 439, 290-294.

Robertson, M., Swain, S.M., Chandler, P.M. and Olszewski, N.E. (1998) Identification of a negative regulator of gibberellin action, HvSPY, in barley. Plant Cell, 10, 995-1007.

Rock, C.D. and Quatrano, R.S. (1995) The role of hormones during seed development. In Plant Hormones (Davies, P. J., ed.). The Netherlands: Kluwer Academic Publishers, pp. 671-697. 
Rogers, J.C., Lanahan, M.B. and Rogers, S.W. (1994) The cis-acting gibberellin response complex in high pl alpha-amylase gene promoters. Requirement of a coupling element for high-level transcription. Plant Physiol. 105, 151-158.

Rubio-Somoza, I., Martínez, M., Abraham, Z., Diaz, I. and Carbonero, P. (2006a) Ternary complex formation between HvMYBS3 and other factors is involved in transcriptional control in barley seeds. Plant J. 47, 269-281.

Rubio-Somoza, I., Martinez, M., Diaz, I. and Carbonero, P. (2006b) HvMCB1, a R1MYB transcription factor from barley with antagonistic regulatory functions during seed development and germination. Plant $J .45,17-30$.

Shen, 0., Gomez-Cadenas, A., Zhang, P., Walker-Simmons, M.K. Sheen, J. and Ho, T.H. (2001) Dissection of abscisic acid signal transduction pathways in barley aleurone layers. Plant Mol. Biol. 47, 437-448.

Steber, C.M. and McCourt, P. (2001) A role for brassinosteroids in germination in Arabidopsis. Plant Physiol. 125, 763-769.

Sun, T.P. and Gubler, F. (2004) Molecular mechanism of gibberellin signaling in plants. Annu. Rev. Plant Biol. 55, 197-223.
Ueguchi-Tanaka, M., Ashikari, M., Nakajima, M. et al. (2005) GIBBERELLIN INSENSITIVE DWARF1 encodes a soluble receptor for gibberellin. Nature, 437, 693-698.

Washio, K. (2001) Identification of Dof proteins with implication in the gibberellin-regulated expression of a peptidase gene following the germination of rice grains. Biochim. Biophys. Acta, 1520, 54-62.

Xie, Z., Zhang, Z.-L., Yang, G., Komatsu, S. and Shen, Q.J. (2006) Interactions of two abscisic-acid induced WRKY genes in repressing gibberellin signaling in aleurone cells. Plant $J .46,231-$ 242.

Zentella, R., Yamauchi, D. and Ho, T.H. (2002) Molecular dissection of the gibberellin/abscisic acid signaling pathways by transiently expressed RNA interference in barley aleurone cells. Plant Cell, 14, 2289-2301.

Zhang, Z.L., Xie, Z., Zou, X., Casaretto, J., Ho, T.H. and Shen, O.J. (2004) A rice WRKY gene encodes a transcriptional repressor of the gibberellin signaling pathway in aleurone cells. Plant Physiol. 134, 1500-1513.

Accession numbers: HvDof2 (AM084350), HvDof8 (AM084356), HvDof17 (AJ969263), HvDof19 (AJ31232), HvDof23-Sad (AJ312297), HvDof24-Pbf (AJ000991), GAMyb (X87690), CatB (AJ310426), Al21 promoter (X66116) and HvActin2 (BQ768853). 